\section{SiN Nanopores Enable Electrical Bar-Code Scanning of Tagged DNA}

As the cost of DNA sequencing has decreased dramatically, genome sequences of numerous pathogens are readily available, and their number is growing daily. However, many pathogens are genetically similar, and differentiating between them has proven to be a difficult process with current methods. To facilitate more timely, economical, and accurate investigation of infectious agents, laboratories around the world are investigating various purely electrical schemes of detecting proteins. In the February 10 issue of Nano Letters (DOI: 10.1021/nl00058y; p. 738), A. Meller and M. FrankKamenetskii, professors at Boston University (BU), and their co-workers have introduced just such a technology: By electrostatically pulling a tagged, doublestranded DNA strand through a nanoscale opening on a chip, they detect changes in ion current that discriminate between tagged and untagged sites.

The researchers milled single 4-5 nm diameter nanopores in 30-nm thick freestanding SiN membranes using focused electron beam lithography. The $20 \mu \mathrm{m}$ by $20 \mu \mathrm{m}$ membrane with the pore is embedded on a $25 \mathrm{~mm}^{2}$ silicon chip that separates two miniature fluid chambers filled with a $1 \mathrm{M}$ solution of $\mathrm{KCl}$. When a positive bias voltage is applied across the membrane, the DNA, which holds a negative charge, threads through the nanopore. This opening is just large enough to allow the molecule to pass through it without bunching (see Figure 1).

Senior research associate H. Kuhn prepared DNA molecules, which contain specific sequences bound to peptide nucleic acid (PNA) probes. As the DNA



Figure 1. Schematic depicts a DNA molecule with two peptide nucleic acid (PNA) probes threaded through a 4-5 nm nanopore in a 30-nm thick free-standing silicon nitride membrane under a voltage bias. Inset: A transmission electron microscope image of a $4 \mathrm{~nm}$ nanopore. Reproduced with permission from Nano Letters 10 (2) (2010) 738; DOl: 10.1021/nl100058y. (c) 2010 American Chemical Society.

threads through the nanopore, the molecules partially block the opening between the chambers which reduces the ion current between them. Because the PNAtagged regions have a greater crosssectional area than the bare DNA, as these sites pass through the membrane they increase the resistance of the nanoscale feature. The resultant change in the current was easily detected in work by graduate students A. Singer and W. Morrison and research associate M. Wanunu. Tests with control samples showed that there were no effects due to non-specific binding. Furthermore, the technique was able to distinguish between once-tagged and twice-tagged samples.

The research team said that given the known sequences, it is possible to develop a bar-coding method with the highly specific PNA, and then readout that barcode with the solid-state nanopore. Through proper tagging, it is possible to create unique single-molecule signatures that are easily identified as they pass through the nanopore. This method would dramatically improve pathogen detection in a diagnostic setting, ultimately improving patients' outcomes.

JIM RANTSCHLER

\section{Study of Mesoscale Epitaxy Using Colloidal Particles Reveals Dynamics Similar to Atomic-Scale Film Growth}

Epitaxial film growth is a widespread and powerful fabrication method in modern materials research. Research into the physical principles that govern the growth of surface films atop a template crystalline substrate has enabled scientists to more effectively predict and design materials that possess desirable properties. In the January 22 issue of Science (DOI: 10.1126/science.1179947; p. 445), researchers at Cornell University report that many of the same rules that govern atomic-scale epitaxial film growth also apply to surface layers grown epitaxially using colloidal particles, although the difference in size scale introduces some mechanistic dif- ferences. Their findings should enable better modeling and prediction tools to be developed for the growing community of metamaterials researchers, and further experiments using their model system may also prove useful to researchers working at the atomic scale. Other applications of these findings may be found in the design of self-assembled nanomaterials or biologically based materials.

In order to quantify the kinetics of epitaxial growth at the microscale, I. Cohen and postdoctoral associate R. Ganapathy, together with graduate students M.R. Buckley and S.J. Gerbode, first prepared substrates with square or triangular lattices by depositing colloidal monolayers atop a lithographically patterned template with artificial lattice spacings of $\sim 1 \mu \mathrm{m}$. Subsequent layers of colloids were sedimented at a controlled rate on top of these substrates while the movement of individual particles and growth of layers were observed microscopically over time.

The researchers tested two colloid systems, consisting of either chargestabilized silica $(1.0 \mu \mathrm{m}$ diameter $)$ or polystyrene colloids (1.3 $\mu \mathrm{m}$ diameter) particles in polymer solutions that introduced interparticle attractive forces by acting as a depletant. In order to compare their experimental results to existing models, the researchers designed their colloidal deposition solutions with diffusion constant: deposition flux $(D / F)$ ratios on the same order as atomic-scale systems.

Using this system, the research team 